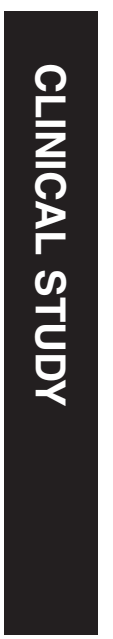

\section{A novel graft option after pterygium excision: platelet-rich fibrin for conjunctivoplasty}

\author{
Abstract \\ Purpose To compare the surgical results, \\ complications, and recurrence rates of primary \\ pterygium excision with conjunctival autografts \\ (CA) vs platelet-rich fibrin (PRF) grafts. \\ Patients and methods A total of 35 eyes of \\ 35 patients with primary pterygium were \\ included in the study. The patients \\ underwent excision of pterygium followed by \\ closure of the bare sclera by CA (group 1 , \\ $n=20$ ) $v$ s PRF grafts (group $2, n=15$ ). The \\ PRF was generated from the patients' own \\ whole-blood sample by centrifugation and \\ pressing. The surgery times, intra/ \\ postoperative complications, recurrence rates \\ of pterygium, and changes in visual acuity \\ (VA) were evaluated and compared within \\ groups. \\ Results The mean follow-up period was \\ $14.3 \pm 6.5$ months (6-24 months). The mean \\ preoperative and postoperative VAs were \\ same $(20 / 25)(P=0.204)$. The mean surgery \\ time was shorter in group $2(22.1 \pm 1.9 \mathrm{~min})$ \\ compared to group $1(33.8 \pm 7.8 \mathrm{~min})$ \\ $(P=0.001)$. The recurrence was observed only \\ in one $(6.6 \%)$ case of group 2 , while none of \\ the cases showed recurrence in group 1. Graft \\ loss was observed in $2(\mathbf{1 0} \%)$ cases in group 1 , \\ and $1(6.6 \%)$ case in group 2 . No other intra/ \\ postoperative complications such as tear in \\ the graft, excessive bleeding, scleral necrosis, \\ graft necrosis, pannus formation, or \\ symblepharon occurred in both groups. \\ Conclusions This preliminary study showed \\ encouraging results of the application of PRF \\ for conjuntivoplasty after pterygium excision. \\ The use of PRF in pterygium surgery is a \\ simple, easily applicable, and a promising \\ method with low rates of recurrence and \\ complications. \\ Eye (2017) 31, 1606-1612; doi:10.1038/eye.2017.109; \\ published online 16 June 2017

\section{Introduction} \\ Pterygium is a common worldwide ocular \\ surface disease, which is a result of abnormal \\ fibrovascular tissue proliferation originates from \\ the degenerative conjunctiva and the underlying \\ tenon. ${ }^{1,2}$ Pterygium is more common in sunny, \\ hot, dry, windy, dusty climate conditions, \\ smoky environments, and tropical regions. 3,4 \\ Ultraviolet radiation is the major risk factor, \\ which damages cell proliferation cycle and \\ induces abnormal cell proliferation, squamous \\ metaplasia, goblet cell hyperplasia, \\ inflammation, fibrosis, angiogenesis, and \\ extracellular matrix breakdown..$^{5}$ Although the \\ pathogenesis of pterygium remains unclear, it is \\ acceptable as a stem cell disorder, which allows \\ extension of the wing-shaped fibrovascular \\ proliferation onto the cornea, and as an \\ epithelia-mesenchymal transition. 6,7 Today, \\ there are many approaches to its treatment \\ including leaving bare sclera, primary \\ conjunctival closure, conjunctival autografts \\ (CA), conjunctival-limbal autografts, and \\ amniotic membrane transplantation (AMT). 5,8,9 \\ Recurrence rates, the major concern after \\ pterygium surgery, within these methods vary \\ widely, therefore, adjunct metabolites like \\ 5-fluorouracil (5-FU), mitomycin-C (MMC), \\ or thiotepa have been used to improve the \\ results. ${ }^{4,8,9}$ However, there exists no \\ surgical technique accepted as the gold \\ standard treatment that prevents \\ recurrence. \\ Platelet-rich fibrin (PRF), described by \\ Choukroun et $a l_{,}^{10}$ is a second-generation \\ platelet concentrate, consists of many growth \\ factors, cytokines, and glycoproteins such as \\ vascular endothelial growth factor, platelet- \\ derived growth factor (PDGF), transforming \\ growth factor- $\beta$, fibronectin, and
}

HB Cakmak ${ }^{1}$, G Dereli Can², ME Can ${ }^{3}$ and $\mathrm{N}$ Cagil $^{2}$ 
thrombospondin, which have key roles in hemostasis and wound healing. ${ }^{11,12}$ Because of PRF's natural fibrin framework properties, growth factors can keep their activity for a relatively longer period and stimulate cell migration, wound healing, and tissue regeneration effectively. ${ }^{12,13}$ Additionally, the PRF provides temporary scaffold function for growing cells to migrate easily on/ into this membrane. ${ }^{14}$ Beside these features, PRF clots can easily be transformed in dense fibrin membranes or cylinders by using suitable tools.

Although the PRF has been used in oral, ear-nosethroat, orthopedic, and plastic surgeries as a biomaterial, it has not been employed for pterygium surgery until now. ${ }^{14}$ We advocated that PRF is a novel autologous biomaterial and a suturable membrane for ocular surface reconstruction after pterygium excision.

The aim of this study was to evaluate the efficacy and safety of PRF grafting in primary pterygium surgery.

\section{Materials and methods}

\section{Subjects}

This was a prospective, comparative, non-randomized clinical trial conducted at the Department of Ophthalmology, Yildirim Beyazit University, Ataturk Training and Research Hospital, Ankara, Turkey, according to the tenets of the declaration of Helsinki. Approval was obtained from the Institutional Review Board of Yildirim Beyazit University Hospital and written informed consent was obtained from all participants after the explanation of the research design.

We prospectively and consecutively enrolled a total of 35 pterygium patients with unilateral nasal primary pterygium infiltrating cornea $2-5 \mathrm{~mm}$ in size, who underwent pterygium excision between June 2013 and September 2014.

The indications for pterygium surgery were as follows: reported growth by patient, prominent vascularization, and intractable symptoms of irritation. Patients with pseudopterygium, infection of the ocular surface or eyelids, a history of prior ocular surgery for pterygium, or systemic diseases that might have an effect on the healing of the surgical site were excluded.

After pterygium diagnosis, details of two surgical techniques were discussed with patients before the operation. Possible peroperative and postoperative risks were explained in detail. PRP is a new surgical method and the possible complications and safety concerns were discussed. Patients were assigned to treatment groups according to their preference after a thorough discussion of each surgical techniques, concerning possible implications and complications. The patients were allocated into two groups: group 1 consists of 20 patients who underwent primary pterygium excision followed by closure of the bare sclera with CA, group 2 consists of 15 patients who underwent primary pterygium excision followed by closure of the bare sclera with PRF graft.

\section{Preparation of the PRF}

Blood samples were taken with a 24-gauge butterfly needle from the patient's antecubital vein, immediately before surgery in the operating room. Samples were collected in $10 \mathrm{ml}$ glass tubes without anticoagulant in the operating room just before the operation. Blood samples were immediately centrifuged at 2.700 r.p.m. for $12 \mathrm{~min}$ using a table centrifuge system (Hettich EBA-20; Hettich Holding GmbH \& Co. oHG, Kirchlengern, Germany). The fibrin clot that formed in the middle part of the tube was removed (Figure 1a), and remnants of red blood cells were scraped off with gauze (Figure 1b). The clot was transferred to a PRF box (Medisoft Medical, Ankara, Turkey) and compressed, and the PRF membranes were obtained (Figure 1c). As soon as PRF membrane was prepared, it was sutured, without any delay.

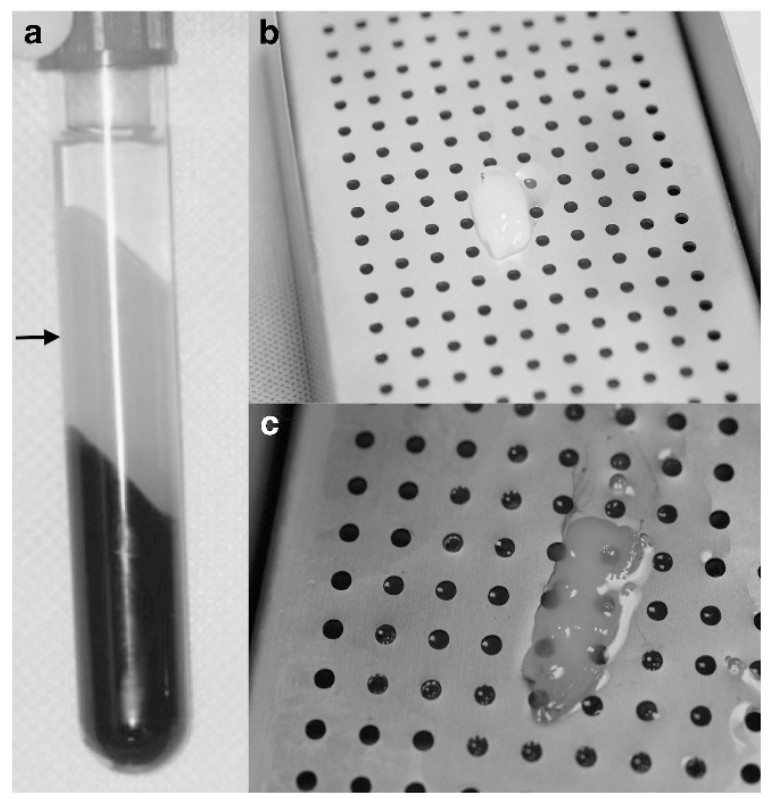

Figure 1 The preparation protocol of the PRF membrane. (a) The fibrin clots were concentrated (arrow) between the red blood cell corpuscles at the bottom and the acellular plasma at the top of the tubes. (b) Separation of the PRF clot from red blood cell layer. (c) The suturable membrane formation prepared from the fibrin clot by pressing in the PRF box. Note the semitransparency and smooth surface of the membrane. 


\section{Surgical technique}

All surgical procedures were performed by one surgeon (GDC) under topical (0.5\% propacaine $\mathrm{HCl}$ : Alcaine; Alcon Inc., Forth Worth, TX, USA) and subconjunctival (lidocaine $10 \mathrm{mg} / \mathrm{ml}+$ epinephrine $0.0125 \mathrm{mg} / \mathrm{ml}$ : Jetokain; Adeka, Samsun, Turkey) anesthesia. After a careful dissection, pterygium head and a small part of its body were excised with the underlying tenon tissue from the cornea and the sclera using scissors. Residual pterygium tissue on the cornea was stripped with crescent knife. In group 1, the bare sclera was measured with caliper, and a same sized CA was harvested from superotemporal bulbar conjunctiva without damaging the limbus, and was placed on the bare sclera. The CA was oriented over the scleral bed in the way that the limbal side in the graft remained limbal, and secured to the surrounding conjunctiva with 4-6 separated 7/0 vicryl sutures (DLZ-6.4-200, FSSB, Jestetten, Germany). In group 2 the PRF, prepared just before the pterygium excision, was trimmed to fit the size of the defect, and placed on the bare sclera. After orientation, it was secured with 4-6 separated 7/0 vicryl sutures (DLZ-6.4200, FSSB, Jestetten, Germany) to the surrounding conjunctiva. The surgery times of both procedures, and complications occurred during the surgery such as excessive bleeding, tears in the grafts, scleral damage, or graft loss were recorded for comparison.

Postoperatively, all subjects received an antibiotic eye drop (0.3\% Ofloxacin: Exocin; Allergan Inc.) four times daily for 1 week, and lubricant eye drop (Polyvinyl Alcohol and Povidone: Refresh single; Allergan Inc., Parsippany, NJ, USA) four times daily for 6 weeks. In addition, corticosteroid eye drop $(0.1 \%$ Fluorometholone and $0.025 \%$ Tetrahydrozoline Hydrochloride: Efemoline; Novartis Ophthalmics AG Hettlingen, Forth Worth, TX, USA) was prescribed three times per day starting from third postoperative day up to 3 weeks.

\section{Follow-up examinations}

After descriptive evaluation of the subjects including age, gender, medical and ocular history, a comprehensive ophthalmic evaluation of all cases including best spectacle corrected visual acuity (BSCVA), refraction, tonometry, slit-lamp biomicroscopy, and dilated fundus examination was performed before and after surgery. In the postoperative period the patients were visited on days 1 , 3, 5, and 1 week, 1 month, 6 months, 12 months, and 24 months after surgery. Preoperative and postoperative slit-lamp examinations in which the grafted area was observed for the presence of injection, pigmentation, granuloma, melting, or infection were documented photographically at each visit in the same room conditions, and the same device settings. Efron scale for conjunctival redness was used to scale degree of postoperative inflammation at the end of 1 month. In this scale conjunctival redness graded as normal, trace, mild, moderate, and severe. ${ }^{15}$ Additionally, conjunctival epithelialization on surgical margins was closely monitored with fluorescein staining. Presence of reaction against sutures evidenced by mucous secretion and excessive hyperemia on conjunctiva was noted.

Recurrence was defined as a fibrovascular growth over the nasal cornea with conjunctival drag extending beyond the surgical limbus.

\section{Statistical analysis}

Data were coded, entered, and analyzed with Statistical Package for the Social Sciences software version 18.0 (SPSS Inc., Chicago, IL, USA). Results were expressed as mean \pm SD. A Mann-Whitney $U$-test was performed to analyze the changes in continuous variables. Categorical variables were tested with Chi-square test. A $P$-value of less than 0.05 is considered statistically significant. Power analysis was performed by using GPower 3.1 software (Dusseldorf, Germany).

\section{Results}

\section{Demographics}

There were $6(30 \%)$ female and $14(70 \%)$ male subjects in group 1, $8(53.4 \%)$ female and $7(46.6 \%)$ male subjects in group $2(P=0.148)$. The mean age of the patients in group 1 and 2 was $49.3 \pm 12.4$ (between 27 and 75) years and $55.2 \pm 13.5$ (between 30 and 70) years, respectively $(P=0.551)$. The mean duration of the pterygium was $5.1 \pm 4.6$ (between 1 and 20) years in group 1 and $5.2 \pm 2.7$ (between 1 and 10$)$ years in group $2(P=0.216)$. The size and morphology of pterygium were similar in both groups.

\section{Visual acuity}

The mean BSCVA was 20/25 preoperatively in group 1 and group 2, and it was not changed 12 months after the surgery in both groups $(P=0.204)$. None of the cases showed decrease in BSCVA.

\section{Surgery time and follow-up examinations}

The average surgical time for group 2 was shorter $(22.1 \pm 1.9 \mathrm{~min})$ when compared to group 1 $(33.8 \pm 7.8 \mathrm{~min})(P=0.001)$. No intraoperative complication was noted for both groups. The mean follow-up period was $14.3 \pm 6.5$ months (6-24 months) for 

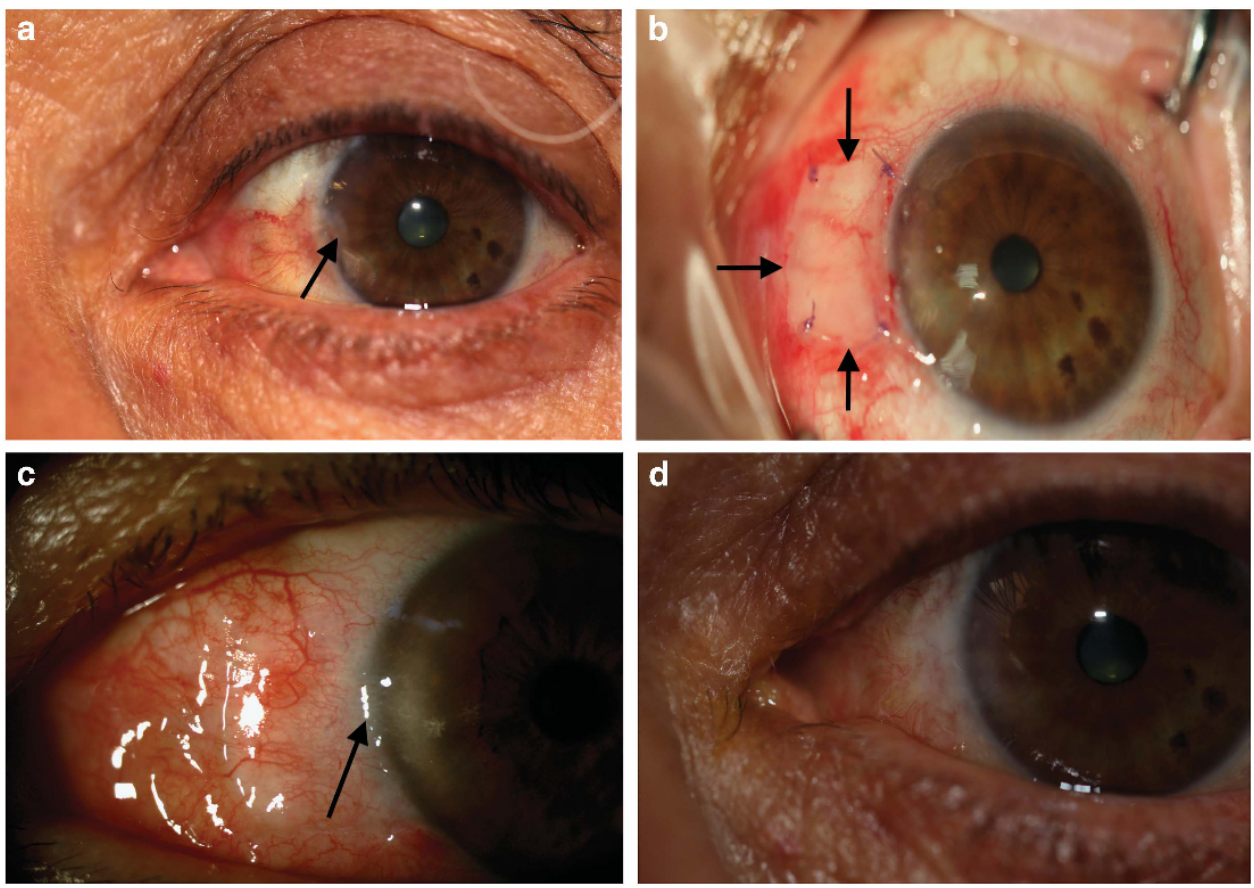

Figure 2 Sequential anterior segment photographs of a case. (a) Primary nasal pterygium, swollen on the limbal site, infiltrates cornea $2 \mathrm{~mm}$ (arrow), and mild-moderate nasal conjunctival hyperemia. (b) PRF secured with separate 7/0 absorbable suture material on the bare sclera. Note the smooth transition between host conjunctiva and the membrane (arrows). (c) Postoperative first week slit-lamp examination. PRF dissolves, conjunctival epithelialization was completed, and limbal site was clear (arrow). (d) Postoperative sixth month slit-lamp examination. There was no recurrence, hyperemia, roughness, or other complications.

both groups $(P=0.5)$. In early postoperative period, slitlamp examination revealed shorter durations of epithelialization of the defective zone in group 1 ( $3.5 \pm 1.3$ days) than in group 2 ( $4.0 \pm 3.1$ days). The difference was not statistically significant $(P=0.681)$. Graft loss was observed in $2(10 \%)$ cases in group 1 and 1 $(6.6 \%)$ case in group 2 on day 5 postoperatively $(P=0.207)$. Delayed epithelialization was also observed in those cases.

Suture reaction was observed in $3(15 \%)$ cases of group 1. In one of those cases, pyogenic granuloma formation was developed at the first postoperative month. Likewise, postoperative inflammation graded as moderate was observed in $6(30 \%)$ cases in group 1. No suture reaction was observed in group 2 and the difference between two groups was not statistically significant with regard to suture reactions $(P=0.117)$. On the contrary, postoperative inflammation scores were significantly lower in group $2(P=0.020)$.

In addition biomicroscopic evaluation revealed excessive mucous secretion in $5(25 \%)$ cases in the early postoperative period, which decreased up to first week in group 1. Whereas, this condition was not observed in any cases in group 2. No other postoperative complications such as scleral necrosis, graft necrosis, pannus formation, or symblepharon occurred in both groups.

On the sixth month postoperative visit, the recurrence was observed only in one (6.6\%) case in group 2 .

Although the perioperative and early postoperative period was free of complications and the healing process proceeded smoothly in that case, the complaints including mild irritation sensation and hyperemia were started 4 months after surgery. A lubricant eye drop (Polyvinyl Alcohol and Povidone: Refresh single; Allergan Inc.) and a corticosteroid eye drop $(0.1 \%$ Fluorometholone and 0.025\% Tetrahydrozoline Hydrochloride: Efemoline; Novartis Ophthalmics AG Hettlingen) were prescribed four times daily for 6 weeks. According to the quiescent results of the biomicroscopic evaluation performed 2 months after the medical treatment, no other procedure was applied to that patient.

Figure 2 shows sequential anterior segment photographs of a case, taken preoperatively, intraoperatively, and on postoperative seventh day and sixth month.

\section{Discussion}

Traditionally, several treatment modalities such as leaving bare sclera, primary conjunctival closure, 
conjunctival with/without limbal auto-grafting, and AMT have been used for ocular surface reconstruction after pterygium excision. ${ }^{4,5,8,9}$ However, the most common challenging problem is recurrence rate after pterygium surgery. ${ }^{16}$ The risk factors of pterygium recurrence are as follows: pterygium type and size, age of patient, environmental conditions, recurrent pterygium, and surgical technique. ${ }^{17}$ Furthermore, surgical trauma, postoperative inflammation, fibroblast proliferation, and deposition of extracellular matrix protein have been related with the mechanism of pterygium recurrence. ${ }^{18,19}$ Therefore, the main successful target of pterygium surgery is to prevent recurrence. To this end, adjunctive anti-mitotic agents such as $\beta$-irradiation, thiotepa, 5-FU, and MMC have been commonly tried with pterygium excision to reduce the rate of recurrence. $4,8,9,20$

Pterygium excision followed by leaving bare sclera is out of favor due to its high recurrence rates. Therefore, CA technique has gradually become a popular treatment for pterygium in the $1980 \mathrm{~s}^{21}$ This technique has been reported to have low recurrence rates. However it has some important disadvantages like requirement of longer operating times, postoperative ocular irritation, and disruption of normal anatomy and microenvironment of the conjunctival tissue. ${ }^{22}$

PRF described by Choukroun et al, ${ }^{10}$ is a secondgeneration platelet concentrate, consists of 3-D polymerized autologous fibrin matrix with the incorporation of platelets, growth factors, cytokines, circulating stem cells, and small amount of leukocytes, which have key roles in hemostasis and wound healing. The intrinsic incorporation of these factors within this scaffold allows for their progressive and controlled release, as the fibrin mesh degrades. PRF releases autologous growth factors gradually, resulting in a stronger and more durable effect on cell proliferation, differentiation, migration, and matrix synthesis by binding to specific cell-surface receptors. ${ }^{10,23}$ Moreover, PRF provided mechanical support to the migrating conjunctival and endothelial cells as a scaffold. The combination of mechanical and chemotactic support of autologous PRF makes it suitable for reconstruction, improvement, and/or maintenance of the ocular surface, and might offer many potential clinical advantages for pterygium surgery when compared to other methods.

To the best of our knowledge, this is the first study investigating and comparing the efficacy, safety, recurrence, and complication rates of the PRF grafting vs CA after pterygium excision. In this current study, surgeries with both techniques resulted similar recurrence rates and graft loss. Recurrence rate in the current study could be interpreted as equal as other studies, and better than some reports like Prabhasawat et $a 2^{24}$ who reported recurrence rate $10.9 \%$ with $\mathrm{AMT}$ and some other reports have presented recurrence rate $8-39 \%$ with CA for primary pterygium. ${ }^{20,22,24-28}$

Another important finding is that the PRF grafting resulted in milder postoperative inflammation, tissue reaction, and granuloma formation. ${ }^{16,19}$ PRF also contains a large quantity of platelet and small amount of leukocyte cytokines, which can play a significant role in the selfregulation of inflammatory and infectious phenomena. ${ }^{29,30}$ For this reason, PRF usage might yield a less pronounced postoperative inflammation. If this is the case, it has other implications beside a more comfortable convalesence period. Marked postoperative inflammation might be a stealth generator of recurrence, inducing cellular activity. Following the same line, these findings provide some support for the conceptual premise that PRF usage might have a great impact on diminishing recurrence rates.

Current study revealed that the mean surgery time was shorter in PRF group than CA group about $10 \mathrm{~min}$. Harvesting CA requires additional surgical steps and needs extra time. Shorter surgery times not only decrease surgery costs but also it supports higher patient satisfaction. In prolonged surgeries, patients feel much more pain, causing their cooperation lost due to decrease in effect of anesthesics. In addition, increase in the amount of surgical manipulations and duration of surgery might provoke a more severe postoperative inflammation. Additionally, PRF grafting does not cause any additional conjunctival tissue damage and helps to preserve normal conjunctival anatomy maximally.

Autologous biomaterials are more superior and preferable than homologous and synthetic materials for reconstruction of the damaged tissues. Although this study compared two autologous technic for conjunctivoplasty, the PRF has more advantages when compared to other technics such as AMT, an allograft, which has the least reaction potential. The preparation of AMT is complicated and rather expensive, which requires a strong tissue banking expertise to prevent inadvertent complications. Moreover, contagion risk of some serious pathogens like hepatitis B and human immune deficiency virus always exists and cannot be excluded even in the presence of very strict procedures and measures. Although, immune reactions against autologous PRF do not arise, stringent preparation measures are mandatory to ensure sterility and to minimize pre and post-surgical infection risks.

Preparation of PRF is easy and can be carried out with few instruments in the operation theater. However, GMP clean room facilities are mandatory for PRF 
preparation in some countries, particularly in European countries.

PRF membrane is a suitable medium for microbial growth. Therefore, there is always a risk of spread of infection and to minimize that risk, freshly prepared membranes must be sutured promptly without any delay.

Freshly prepared PRF, even though has some similarities to conjunctiva or amniotic membrane, behaves in a different way during handling and suturing. In the learning phase, inadequate membrane suturing may cause membrane loss. In this study, membrane loss occurred in 1 case (case 5) (6.6\%), in which delayed epithelialization and healing were observed in contrast to other cases.

This study is a preliminary experience with the use of an autologous PRF for conjunctivoplasty after pterygium excision. Although healing responses of cases were impressive and recurrence rate was very low, some important limitations of this study must be taken into account. First, this study is an initial study and only a limited number of cases were included. A limited statistical power because of the modest sample size in the present study $(n=35)$ has a limiting role in significance of the statistical comparisons. A post hoc power analysis, with an observed effect size (0.267), resulted that an $n$ of $\sim 180$ would be needed to obtain statistical power at the recommended 0.80 level. Results of this study, considering its insufficient sample size and inadequate power, must be interpreted with due skepticism. Future randomized studies, employing larger sample sizes, and powerful enough to reach a robust decision, will yield more steadfast conclusions.

Second, in the current study PRF grafting compared with only one autologous method and differences between other alternative methods remains to be compared. Third, patients with primary pterygium were enrolled in this study and results cannot be generalized to the cases with recurrent pterygium. Finally, long-term results are important when making choices among different alternative therapies, and this study covers only a mean of 14 months follow-up period.

As a result, this initial study showed encouraging results and gave a positive impression that PRF grafting in pterygium surgery was a novel, simple, easily applicable, and a promising method with low rates of recurrence and complication. Prospective long-term follow-up studies that additionally compare other techniques with the PRF in larger case groups are required to understand the advantages of PRF grafting in pterygium surgery.

\section{Summary}

What was known before

- Conjunctival autografts, conjunctival-limbal autografts, and amniotic membrane are the most preferred graft materials after pterygium excision.

- Platelet-rich fibrin (PRF) has been used in oral, ear-nosethroat, orthopedic, and plastic surgeries as a graft biomaterial, however, it has not been employed for pterygium surgery until now.

What this study adds

- PRF grafting after pterygium excision is a simple, easily applicable, and a promising method with low rates of recurrence and complications.

- PRF is a novel autologous biomaterial and a suturable membrane for ocular surface reconstruction after pterygium excision.

\section{Conflict of interest}

The authors declare no conflict of interest.

\section{Acknowledgements}

We would like to thank Duygu Kiliç for her help and advice on statistical analysis.

\section{References}

1 Di Girolamo N, Chui J, Coroneo MT, Wakefield D. Pathogenesis of pterygia: role of cytokines, growth factors, and matrix metalloproteinases. Prog Retin Eye Res 2004; 23: 195-228.

2 Kim KW, Park SH, Kim JC. Fibroblast biology in pterygia. Exp Eye Res 2016; 142: 32-39.

3 Li L, Zhong H, Tian E, Yu M, Yuan Y, Yang W et al. Five-year incidence and predictors for pterygium in a rural community in China: the Yunnan minority eye study. Cornea 2015; 34 (12): 1564-1568.

4 Serghiou S, Patel CJ, Tan YY, Koay P, Ioannidis JP. Fieldwide meta-analyses of observational associations can map selective availability of risk factors and the impact of model specifications. J Clin Epidemiol 2016; 71: 58-67.

5 Chui J, Di Girolamo N, Wakefield D, Coroneo MT. The pathogenesis of pterygium: current concepts and their therapeutic implications. Ocul Surf 2008; 6: 24-43.

6 Hirst LW, Axelsen Ra, Schwab I. Pterygium and associated ocular surface squamous neoplasia. Arch Ophthalmol 2009; 127: 31-32.

7 Ando R, Kase S, Ohashi T, Dong Z, Fukuhara J, Kanda A et al. Tissue factor expression in human pterygium. Mol Vis 2011; 17: 63-69.

8 Mahdy MES, Bhatia J. Treatment of primary pterygium: role of limbal stem cells and conjunctival autograft transplantation. Oman J Ophthalmol 2009; 2: 23-26.

9 Taylan Sekeroglu H, Erdem E, Dogan NC, Yagmur M, Ersoz $\mathrm{R}$, Dogan A. Sutureless amniotic membrane transplantation 
combined with narrow-strip conjunctival autograft for pterygium. Int Ophthalmol 2011; 31: 433-438.

10 Dohan DM, Choukroun J, Diss A, Dohan SL, Dohan AJ, Mouhyi J et al. Platelet-rich fibrin (PRF): a second-generation platelet concentrate. Part I: technological concepts and evolution. Oral Surg Oral Med Oral Pathol Oral Radiol Endod 2006; 101: e37-44.

11 Prakash S, Thakur A. Platelet concentrates: past, present and future. J Maxillofac Oral Surg 2011; 10: 45-49.

12 Clark RA. Fibrin and wound healing. Ann NY Acad Sci 2001; 936: 355-367.

13 Öncü E, Alaaddinoğlu E. The effect of platelet-rich fibrin on implant stability. Int J Oral Maxillofac Implants 2015; 30: 578-582.

14 Dohan Ehrenfest DM, Doglioli P, de Peppo GM, Del Corso M, Charrier J-B. Choukroun's platelet-rich fibrin (PRF) stimulates in vitro proliferation and differentiation of human oral bone mesenchymal stem cell in a dose-dependent way. Arch Oral Biol 2010; 55: 185-194.

15 Efron N (eds). Contact Lens Practice, 2nd ed. ButterworthHeinemann: Oxford, UK, 2010.

16 Said DG, Faraj La, Elalfy MS, Yeung A, Miri A, Fares U et al. Intra-lesional 5 fluorouracil for the management of recurrent pterygium. Eye (Lond) 2013; 27: 1123-1129.

17 Alpay A, Uğurbaş SH, Erdoğan B. Comparing techniques for pterygium surgery. Clin Ophthalmol 2009; 3: 69-74.

18 Kwon SH, Kim HK. Analysis of recurrence patterns following pterygium surgery with conjunctival autografts. Medicine (Baltimore) 2015; 94(4): e518.

19 Ling S, Li Q, Lin H, Li W, Wang T, Ye H et al. Comparative evaluation of lymphatic vessels in primary versus recurrent pterygium. Eye (Lond) 2012; 26(11): 1451-1458.

20 Tsim NC, Young AL, Jhanji V, Ho M, Cheng LL. Combined conjunctival rotational autograft with $0.02 \%$ mitomycin $C$ in primary pterygium surgery: a long-term follow-up study. Br J Ophthalmol 2015; 99(10): 1396-1400.
21 Kenyon KR, Wagoner MD, Hettinger ME. Conjunctival autograft transplantation for advanced and recurrent pterygium. Ophthalmology 1985; 92: 1461-1470.

22 Hall RC, Logan AJ, Wells AP. Comparison of fibrin glue with sutures for pterygium excision surgery with conjunctival autografts. Clin Exp Ophthalmol 2009; 37: 584-589.

23 Lee HJ, Choi BH, Jung JH, Zhu SJ, Lee SH, Huh JY et al. Maxillary sinus floor augmentation using autogenous bone grafts and platelet-enriched fibrin glue with simultaneous implant placement. Oral Surg Oral Med Oral Pathol Oral Radiol Endod 2007; 103: 329-333.

24 Prabhasawat P, Barton K, Burkett G, Tseng SC. Comparison of conjunctival autografts, amniotic membrane grafts, and primary closure for pterygium excision. Ophthalmology 1997; 104: 974-985.

25 Kurna SA, Altun A, Aksu B, Kurna R, Sengor T. Comparing treatment options of pterygium: limbal sliding flap transplantation, primary closing, and amniotic membrane grafting. Eur J Ophthalmol 2013; 23: 480-487.

26 Kocamis O, Bilgec M. Evaluation of the recurrence rate for pterygium treated with conjunctival autograft. Graefes Arch Clin Exp Ophthalmol 2014; 252: 817-820.

27 Kaufman SC, Jacobs DS, Lee WB, Deng SX, Rosenblatt MI, Shtein RM. Options and adjuvants in surgery for pterygium: a report by the American Academy of Ophthalmology. Ophthalmology 2013; 120: 201-208.

28 Tsim NC, Young AL, Jhanji V, Ho M, Cheng LL. Combined conjunctival rotational autograft with $0.02 \%$ mitomycin $\mathrm{C}$ in primary pterygium surgery: a long-term follow-up study. Br J Ophthalmol 2015; 99: 1396-1400.

29 El-Sharkawy H, Kantarci A, Deady J, Hasturk H, Liu H, Alshahat $\mathrm{M}$ et al. Platelet-rich plasma: growth factors and pro- and anti-inflammatory properties. J Periodontol 2007; 78: 661-669.

30 Naik B, Karunakar P, Jayadev M, Marshal VR. Role of platelet rich fibrin in wound healing: a critical review. J Conserv Dent 2013; 16: 284-293. 